\title{
Corpus-Aided Teaching Practice on the Depth of CET-4 Vocabulary Knowledge
}

\author{
Feng Minmin
}

\author{
School of General Education, Xi'an Eurasia University, Xi'an, Shaanxi, China \\ fengminmin@eurasia.edu
}

\begin{abstract}
With massive storage of language information, high-speed and stable computer retrieval mode, intuitive and mass operation of language observation interface, corpus linguistics has made great influence on college English teaching in the context of educational informationization. Corpus-aided language teaching would be an effective way in improving students' participation in class activities, enhancing learning effects and promoting the development of students' learning ability. This article introduces the way of compiling of a small corpus and demonstrates the strategies and teaching practice on CET-4 vocabulary knowledge by using the corpus retrieval tool Antconc 3.5.8 $\mathrm{w}$ and the interactive teaching software Hot potatoes 6.3.0.3, aiming to provide feasible and observable methods for teaching CET-4 vocabulary knowledge from different perspectives.
\end{abstract}

Keywords : Deep teaching of vocabulary knowledge; corpus-aided English teaching; interactive teaching

\section{语料库辅助的大学英语四级词汇深度教学实践探索}

\author{
冯敏敏
}

西安欧亚学院通识教育学院, 西安, 陕西, 中国

fengminmin@eurasia.edu

\section{摘要}

语料库语言学作为现代语言学、计算机科学与教育学跨学科互动的新兴学派, 以其前所未有的巨量语 言信息储备、高速精准的计算机检索方式和直观的批量化语言观察界面取胜，对教育信息化背景下的 大学英语教学有巨大的促进和引导作用。使用语料库辅助英语课堂教学是提升学生参与度、增强学生 学习效果、促进学生学习能力发展的有效途径。本文阐述了如何自建英语四级语料库, 并结合语料库 检索工具 Antconc 3.5.8w 和互动式教学软件 Hot potatoes 6.3.0.3, 介绍了大学英语四级词汇深度 教学可采用的策略和教学实践活动, 为多层次词汇深度知识教学提供可操作、可观察的方法。

关键词: 词汇深度教学，语料库辅助英语教学，互动式教学

\section{1. 引言}

语料库及其检索工具为英语词汇教学提供了新思 路和方法, 其理论依据主要参照建构主义理论及图式理 论。语料库既可以作为教师的教学资源, 也可以作为学 生直接学习的资源。在英语词汇教学中的作用主要体现 在以下几个方面: 能够更好地发挥学生在课堂的主体地 位, 对于真实语言材料的分析有利于促进学生利用语料 库进行数据驱动的自主性探究学习能力的提升; 教师可 结合学生的实际需求利用语料库中的资源进行更加科 学合理的教学计划, 对语料库中的语言实例进行详细分 析, 最终实现语言的高质量教与学; 可以增加课堂信息
量的输入, 最大限度提升课堂教学效率。

考虑到国外主要的单语语料库 COCA 和 BNC 语料范 围较大，国内的单语语料库受限于版权、实效性和针对 性等方面的问题, 本文采用自建四级语料库的方式, 尝 试说明语料库在辅助英语四级词汇深度教学领域所发 挥的作用。

\section{2. 基于自建四级教学语料库的数据检索}

\section{1. 研究语料}

自建四级教学语料库的步骤如下: 
1）从网上下载改革之后的大学英语四级考试历年 真题 word 版 (2016.6-2019.12), 经与官方提供的四 级真题纸质版一一核对, 该电子版真题真实有效。下载 网 址 为 https://www.hjenglish.com/sijidaan/ zuixinsijizhenti/。

2) 对下载后的真题进行校队和排版调整，保证原 始语料的准确性。将每一套真题另存为文本文档并重新 命 名 ( 20160601.txt, 201602.txt, 20161201.txt ...20191203.txt)，存放在统一的文件夹 里。

至此, 自建的四级教学语料库就初步形成了。该语 料库的总体规模为类符数 9083 , 形符数 126935。语 料涵盖四级听力、阅读、翻译和写作四类文本材料, 主 要收集了 2016 年 6 月至 2019 年 12 月的四级考试真 题。随着语料库建设的开展, 语料涵盖范围将进一步扩 大，体现语料库的历时性。

\section{2. 研究工具}

\subsection{1. 语料检索软件}

因为 “语料库本身不能做任何事情, 只不过是存储 了一些使用过的语言。而只有与语料库检索工具相结合 才可以对语料进行重新排列并对语言现象进行研究” [4]。本文中语料库检索软件使用的是 antconc 3.5.8 ${ }^{[1]}$, 该软件界面清晰, 操作简单, 完全以研究者的视角进行 规划和界面布局。Antconc 软件是由日本早稻田大学科 学工程学院科学工程英语教育中心的 Laurence Anthony 博士研发的一款免费语料库检索工具, 适合处 理不超过 $200 \mathrm{MB}$ 的英语语料, 运行环境为 Windows 98/windows 10/2000 ME/XP Vista/Mac IOS。

Antconc 有七大功能, 分别是: concordance (语 境共现索引)、concordance plot（索引定位）、file view (文件查看)、clusters（词簇表）、collocates（搭配 词）、word list（词频表）、keyword list（关键词表）。 使用该检索软件可以实现基于语料库和数据驱动的语 言学习教学和研究。antconc 3.5.8w 其界面见图 1 。

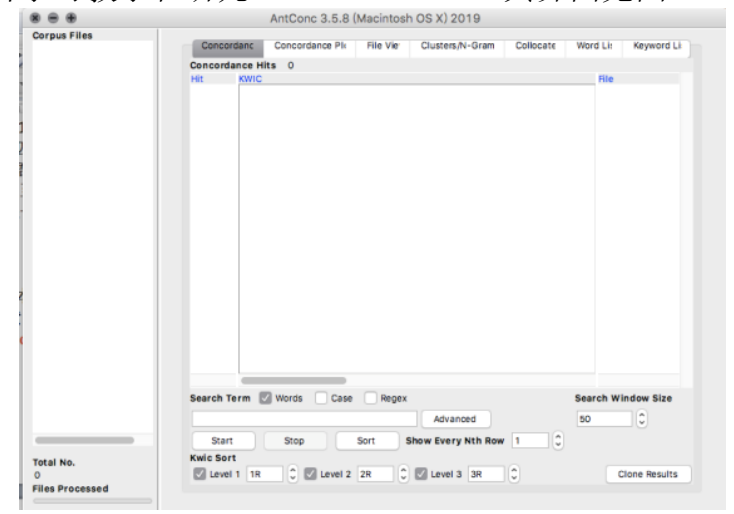

图 1 Antconc 检索软件主界面

\subsection{2. 交互式课件设计软件}

语料库可以用于考察语言的不同方面, 比如句长、 词频、词表、搭配、衔接与连贯等, 并可以对语言进行 共时和历时性的研究。但语料库进入课堂教学需要考虑 教学文件设计、教学活动的开展、课堂实施步骤及评价 手段等内容。对于希望使用语料库数据的教师来说, 主 要的挑战是如何将数据驱动学习从语料库中整合到一 个普通课程的计划中。本文结合互动式教学软件 hot potatoes 6.3.0.3, 具体阐述如何借助该软件将语料库中 的语料检索结果应用于英语四级词汇教学实践中。

Hot potatoes 6.3.0.3 是加拿大 University of Victoria Humanities Computing and Media Centre 研发 小组开发出来的互动式教学软件, 运行环境为 Windows 98/windows 10/ME/NT4/2000/XP/Vista/Mac IOS。该软件可以帮 助教师使用 HTML 和 JavaScript 制作出五种不同类型的 以教育为目的的互动练习题, 包括 JCloze (填空练习题)、 JQuiz (简答练习题)、JCross (纵横字谜练习题)、JMix (重组句子练习题)、JMatch (匹配练习题)。其免费 下 载网 址 https://www.onlinedown.net/soft/6835.htm

\section{3. 提取分析检索项的语境共现}

在语料库检索软件 Antconc 3.2.4w 中打开要处理 的语料, 在 Search Term 框中输入要检索的特定词, 点 击 Concordance 就可以进行语境共现检索了。以 “associate” 为例, 在语料库中进行单项检索时, 检索 到的索引数是 2 。如果想要检索 “associate” 的各种曲 折变化形式在语料库中的使用语境, 则可以使用通配符 在 Search Term 框中输入“associat*”的方式进行检索， 最终检索到的索引数是 26 (如图 2)。

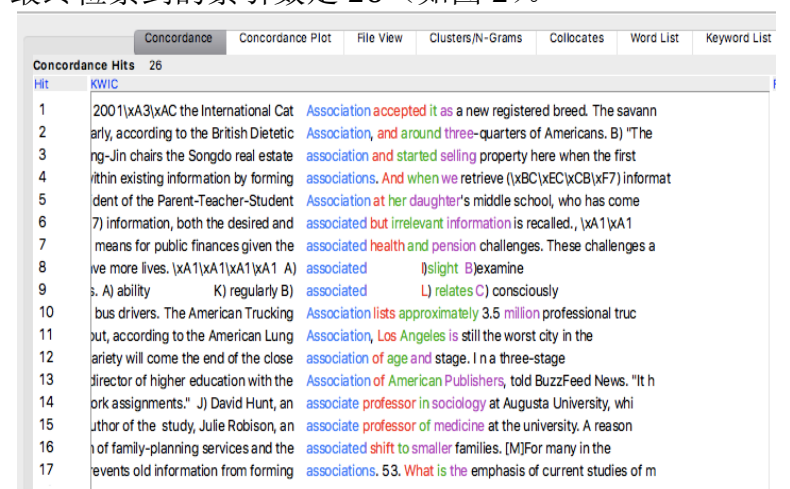

图 2 检索 associat*的语境共现行部分结果

语境共现是某一检索项在指定语料中所出现的语 境共现行的列表 ${ }^{[2]}$ 。与以往教学语例呈现不同的是，它 呈聚焦性排列, 即以检索项居中(如图 2 中的 associat*), 同时呈现其两边局部范围内的语境。通过批量观察分析 这些局部语境可发现检索词项在该语料中的使用特征。 选择一个具体语例, 然后点击 File View (源文件汶览), 
即可看到被选语例的具体语境。

\section{3. 基于自建四级教学语料库的数据分析研 究}

提取上传所有带 associate 的语例, 然后设置 Kwic Sort 中 Level 1=1L, 以凸显 associat*前面的词（如图 3)。 观察它们可以在语法上归纳出多少种词性, 再将各种词 性与 associat*放在一起观察, 看看能够得出 associat* 有多少种词性和意义。有些语例可能需要向前观察, 通 过更多的语境信息来做判断。

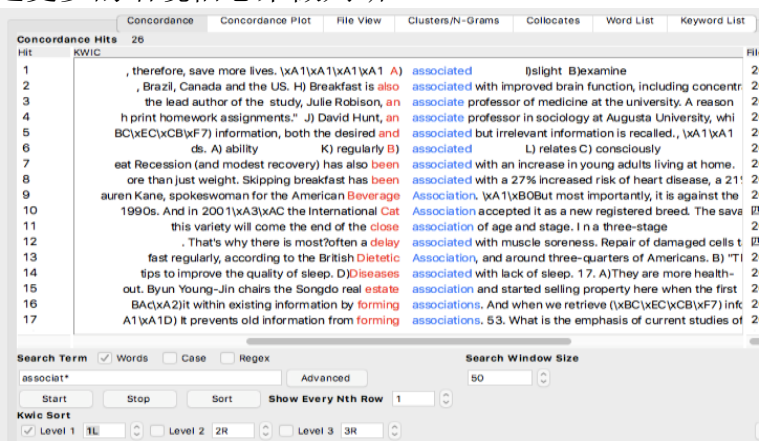

图 3 凸显 associat*左一位置的搭配词

进一步凸显 associat*右一（1R）列的搭配词, 继 续归纳该列词性的特点, 然后结合上一步的发现, 整合 associat*作为各种词性的使用模式。

针对上述的显示结果, 可提取 associat*左一和右 一的词频表, 然后观察期中较高频的搭配词有哪些, 并 进一步分析其后使用介词 of 和 with 时词意是否有区 别。

\section{4. 教学应用}

学生的学习需要在一定情境下进行意义建构, 语料 库能够提供意义建构所需要的语境因素, 这样的语境共 现可以减少学习量, 帮助其在语言使用中培养个人创造 力 ${ }^{[6]}$ 。与此同时, 学生在接受如何观察语言及如何概况 归纳知指导时, 会产生更多的自主性 ${ }^{[3]}$ 。而教师则是为 学习者提供发展他们学习策略的环境, 通过这些策略, 学生可以学到如何学习, 达到大学英语教学的目的之一 ---发展学生学习能力 (大学英语教学指南)。基于语料 库提供的批量语例, 教师可以结合使用 Hot potatoes 6.3.0.3 互动式教学软件制作各类教学微本。

Hot Potatoes 不但操作简便, 而且可以快捷地制作 出网页式的阅读、完型、字谜、配对、排序、简答等不 同形式的练习题, 便于教师应用于教学过程。教师开发 的网上练习题能够让学生不受时间和地点限制, 在互联 网上进行人机交互学习, 而且能够得到及时反馈和自动 评分。

以 associat*为检索项提取所有语例, 将其楛贝到 Hot potatoes 软件中 JCloze 界面下, 进行挖空设置, 然
后保存文件至本地电脑, 即可在课堂上引导学生进行针 对性词汇填空练习, 加深学生对该词汇使用方法的认知。 完型填空题在软件中称为 JCloze, 完成以后的网页习题 与传统的完形填空相似, 学生可直接在文本的空白处键 入单词或短语, 填充完成提交后便可对各道题自动评分。 JCloze 题型通常应用于语法、词汇等练习中。如果将多 个正确答案同时输入至软件中, JCloze 题还可以提供一 题多解, 并对学生的答题自动识别并评分。

配对练习 (JMatch) 有两种样式: 鼠标拖曳式和下 拉选项式。在练习左边选项中插入图片或单词, 便可做 成图片与单词、单词与单词的配对练习。此练习适用于 学生的词汇训练, 可根据需要制作成多义词、反义词、 词组等的搭配练习,让学生在练习的同时逐渐增加自己 的词汇量。

网页式简答题（JQuiz）除了可以帮助教师制作单 选、多选、正误判断等形式的客观选择题, 还可生成主 观测试题。教师只需输入所有可能的答案, 便可对学生 回答的问题进行自动评分, 并显示正确答案或部分正确 答案。

教师完成练习题的制作和存档后, 点击编辑页面上 的网页储存按钮就可将习题存为 HTML 网页形式, 而 无需掌握 HTML 网页制作和 JavaScript 编程技术。网页 习题自动生成后, 只需上传至网站便可供学生课前或课 后的学习和巩固练习。

Hot Potatoes 还具有多媒体应用功能, 可根据需要 在页面插入图片、音频、视频及其它链接等, 让学生边 听、边看、边理解、边练习, 实现学习过程的多维化。 采用真实的多媒体学习材料不仅能够提高教学的趣味 性, 同时还能培养学生的学习策略能力, 这是培养学生 终身学习能力和素质教育的一个重要组成部分。

Hot Potatoes 以多种形式的习题、多媒体材料的应 用以及自动应答和提示功能达到人机互动性的优势, 大 大缩短了刺激一反应的时间, 有利于学生对知识的巩固、 提高学习效率, 也有利于引导语言学习的个性化, 开放 式发展和培养学生自主学习能力。

\section{5. 总结}

教师可以利用语料库对不同语域的词频实施差异 性调查和整理, 将各种语域中较为常见的词句、短语等 进行高效的整理和总结, 有助于英语教师在课堂中对词 汇进行对比性教学 ${ }^{[5]}$ 。同时教师在对语料库的纠正反馈 功能高效利用过程中可以很大程度上提升学生对特定 词汇的正确使用能力, 辅助学生量程良好的英语语言使 用习惯, 促进学生主动认知能力的提升。在教学过程中 结合使用 Antconc 和 Hot Potatoes, 可以帮助教师利用 来自于现实世界的真实语言材料, 开展有趣的、富于交 互性的学习活动, 以实现传统教学模式向新的多媒体教 学模式的转换, 并能增加学习的趣味性, 激发学生的学 习动力, 进而帮助学生提高自主学习能力, 使其充分发 挥自觉意识, 从而把 “有限” 变成 “无限”, 真正作为 学习的主体进行学习。 


\section{REFERENCES}

[1] Anthony, L. (2019). Antconc (Version 3.5.8w) [Computer Software]. Tokyo, Japan: Waseda University. http://www.antlab.sci.waseda.ac.jp/

[2] Baker, P., Hardie, A., McEnery, A. (2006) A Glossary of Corpus Linguistics. University Press, Edinburgh.

[3] Gavioli L, Aston G. (2001) Enriching reality: language corpora in language pedagody. Elt Journal, 55: 238-246.

[4] Susan, H. (2006) Corpora in Applied Linguistics. Beijing World Publishing Corporation, Beijing.

[5] He Anping. (2017) Introduction to Corpus-aid Language Teaching. Foreign Language Teaching and Research Press, Beijing.

[6] Xu Jianbo. (2020) The Application of Corpus in English Teaching and Research in the Context of Big Data. Education Review, 2: 34-35. 